\title{
Impact of BAL lymphocytosis and presence of honeycombing on corticosteroid treatment effect in fibrotic hypersensitivity pneumonitis: a retrospective cohort study
}

\author{
To the Editor:
}

According to a survey study conducted by WIJSENBEeK et al. [1], 76\% of respiratory physicians believe fibrotic hypersensitivity pneumonitis (fibrotic HP, fHP) should be treated with corticosteroids (CS) as first line treatment. However, data to support such a strategy are limited and confined to acute farmer's lung [2]. Classically, HP patients are classified according to symptom chronicity in acute and chronic HP [3]. Based on new data, however, a stratification according to the (radiological) presence of fibrosis seems more in line with prognosis [4]. In an earlier study, we demonstrated that CS treatment was only beneficial in non-fibrotic HP while CS was not effective in fHP, both in terms of survival, and decline in forced vital capacity (FVC) and diffusing capacity of the lung for carbon monoxide ( $\left.D_{\mathrm{LCO}}\right)$ [5]. In the present study, we determined whether the presence of bronchoalveolar lavage lymphocytosis (BAL lymphocytosis, BALL) or honeycombing influences the treatment effect of CS in fHP patients.

We included all fHP patients followed in the University Hospitals Leuven between 2005 and 2016. Patients without BAL were excluded. Validation of HP diagnosis was performed using symptoms, computed tomography (CT) findings and pathological findings as described previously [5]. As only fibrotic patients were included, CT scans were reviewed for the presence of extensive reticulation, traction bronchiectasis or honeycombing (as defined by the Fleischner Society [6]). BALL was dichotomised as "high" when $>20 \%$ and "low" when $\leqslant 20 \%$. We analysed the survival effect of both BALL and honeycombing presence using a Cox proportional hazards model corrected for age, gender and baseline FVC. The effect of CS treatment on pulmonary function was analysed using mixed models comparing FVC $\%$ and $D_{\text {LCO }} \%$ trajectories before treatment initiation with post-treatment FVC \% and $D_{\mathrm{LCO}} \%$ trajectories. CS use was included both with and without time-dependent covariate. Age, gender, active exposure and use of second line immunosuppressive treatment (the latter as time-dependent covariate) were included as fixed effect. Subject and the pulmonary function test-performing hospital were included as random effects (the former as random intercept and independent random slope, the latter as random intercept). Pulmonary function tests were included from 5 years before treatment initiation until 1 year after initiation or until treatment interruption.

$91 \mathrm{fHP}$ patients were included in the study. Mean \pm SD age was $64.6 \pm 11.9$ years, 32 patients (35.2\%) were female. Baseline FVC and $D_{\text {LCO }}$ were $73.6 \pm 21.7 \%$ and $46.7 \pm 17.8 \%$, respectively. 67 patients $(73.6 \%)$ were treated with CS, 20 patients $(21.9 \%)$ received concomitant second line immunosuppressive drugs; 36 patients (40\%) had high BALL, 55 had low BALL (60\%); 58 patients (64\%) had no honeycombing, 33 patients (36\%) had honeycombing. Low BALL was associated with increased honeycombing presence (low versus high BALL: $50.9 \%$ versus $13.9 \%, \mathrm{p}<0.001)$. No other differences in baseline characteristics were noted between low and high BALL patients. Honeycombing presence was associated with decreased BALL (honeycombing present versus absent: $11.8 \%$ versus $24.1 \%, \mathrm{p}=0.002)$ and lower $D_{\mathrm{LCO}}(40.7 \%$ versus $49.8 \%$, $\mathrm{p}=0.025)$. No other differences in baseline characteristics were noted between patients with and without

@ERSpublications

Low BAL lymphocytosis and presence of honeycombing predict poor outcome and absence of corticosteroid treatment effect in fibrotic hypersensitivity pneumonitis http://bit.ly/2QU10K6

Cite this article as: De Sadeleer LJ, Hermans F, De Dycker E, et al. Impact of BAL lymphocytosis and presence of honeycombing on corticosteroid treatment effect in fibrotic hypersensitivity pneumonitis: a retrospective cohort study. Eur Respir J 2020; 55: 1901983 [https://doi.org/10.1183/13993003.01983-2019]. 
honeycombing. Exposure type (mold versus birds versus other versus unknown exposure) was not associated with BALL $(p=0.37)$, honeycombing presence $(p=0.21)$ or death $(p=0.2)$.

Both low BALL and honeycombing presence were associated with poor 10-year survival (low BALL, HR 2.66, 95\% CI 1.05-6.73, p=0.038; honeycombing presence, HR 3.80, 95\% CI 1.66-8.73, $\mathrm{p}=0.002$ ) as depicted in figure $1 \mathrm{a}$ and $\mathrm{b}$. In a Cox proportional hazards model including both BALL and honeycombing presence (corrected for age, gender and baseline FVC \%), only honeycombing was associated with poor outcome (honeycombing, HR 2.68, 95\% CI 1.02-7.06, p=0.046; BALL, HR 1.4, 95\% CI $0.51-4.09, \mathrm{p}=0.49)$. Patients with high BALL experienced an FVC increase of $5.66 \%(\mathrm{p}=0.004)$ after CS initiation, although FVC decline (i.e. the slope of the mixed model) was similar $(-6.6 \%$ per year before versus $-5.3 \%$ per year after CS initiation, $\mathrm{p}=0.77$ ). No effect on $D_{\mathrm{LCO}}$ was observed (main effect (i.e. the intercept): $\mathrm{p}=0.29$, decline (i.e. slope of the model): $\mathrm{p}=0.50$ ). No CS effect was seen in the low BALL group (FVC \% main effect: $\mathrm{p}=0.93$; FVC $\%$ decline: $\mathrm{p}=0.96 ; D_{\mathrm{LCO}} \%$ main effect: $\mathrm{p}=0.50 ; D_{\mathrm{LCO}} \%$ decline: $\mathrm{p}=0.33)$. Patients without honeycombing experienced a trend towards FVC increase of $4.21 \%(\mathrm{p}=0.07)$ after CS initiation, although FVC decline was not affected $(-1.4 \%$ per year before versus $-2.82 \%$ per year after CS initiation, $\mathrm{p}=0.78$ ). No effect on $D_{\mathrm{LCO}}$ was observed (main effect: $\mathrm{p}=0.12$, decline: $\mathrm{p}=0.91$ ). No CS effect was seen in the group with honeycombing (FVC \% main effect: $p=0.80$; FVC $\%$ decline: $p=0.57$; $D_{\text {LCO }} \%$ main effect: $\mathrm{p}=0.33 ; D_{\text {LCO }} \%$ decline: $\left.\mathrm{p}=0.24\right)$. Pulmonary function trajectories are depicted in figure $1 \mathrm{c}-\mathrm{j}$.
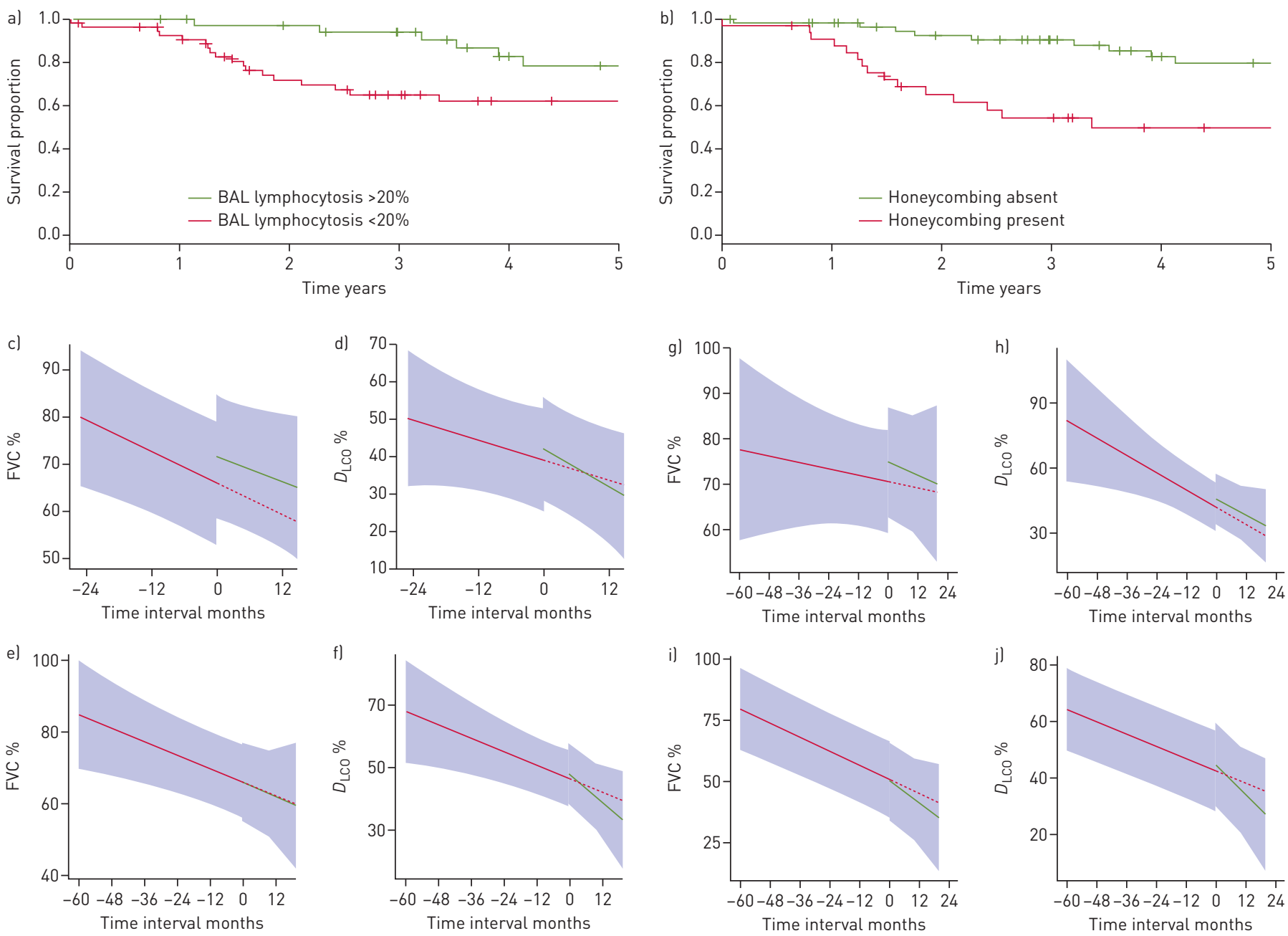

FIGURE 1 Impact of bronchoalveolar lavage (BAL) lymphocytosis and honeycombing in fibrotic hypersensitivity pneumonitis. a) Impact of BAL lymphocytosis on survival. b) Impact of honeycombing on survival. Impact of BAL lymphocytosis (c-f) and honeycombing (g-j) on pulmonary function test trajectories. The solid red line represents the pre-treatment trajectory, the green line represents the post-treatment trajectory, the dotted red line represents the pre-treatment trend. c) Forced vital capacity (FVC) trajectory in high BAL lymphocytosis patients, d) diffusing capacity of the lung for carbon monoxide $\left(D_{\mathrm{LCO}}\right)$ trajectory in high BAL lymphocytosis patients, e) FVC trajectory in low BAL lymphocytosis patients, f) $D_{\text {LCo }}$ trajectory in low BAL lymphocytosis patients, g) FVC trajectory in patients without honeycombing, h) $D_{\text {Lco }}$ trajectory in patients without honeycombing, i) FVC trajectory in patients with honeycombing, and j) $D_{\text {Lco }}$ trajectory in patients with honeycombing. 
While CS initiation had a marginal effect on FVC when BALL was high and when honeycombing was absent, no effect was seen when BALL was low or when honeycombing was present. However, this single centre retrospective study has some limitations. As BALL and honeycombing were correlated in this cohort of 91 patients, there was a lack of statistical power to assess the differential contribution of both variables on FVC increase after treatment initiation. Hence, it is unclear whether the increase of FVC in patients without honeycombing is attributable to the absence of honeycombing or the associated presence of high BALL. Larger multicentre trials are needed to address this question.

As no generally accepted cut-off for high BALL exists, we opted for the cut-off used in our interstitial lung disease (ILD) clinic (i.e. 20\%). Moreover, analysis of c-indices showed a $20 \%$ cut-off yielded the best prognostic separation (c-index 0.635); better compared to a $25 \%$ cut-off (c-index $0.59, \mathrm{p}=0.029$ ), a $30 \%$ cut-off (c-index 0.60, p=0.084) and a 40\% cut-off (c-index 0.51).

We strongly believe that the risks of initiating CS therapy should be balanced against the marginal gains in the subgroup with high BALL/no honeycombing. The recently published nintedanib trial in progressive fibrosing ILDs (PF-ILDs) [7] complicates the decision to initiate CS in fHP patients with high BALL or absence of honeycombing even more. In order to assess whether fHP patients are progressive (which would indicate antifibrotics are warranted), a careful observation of FVC decline of at least $5 \%$ is warranted, based on the nintedanib trial [7]. The marginal increase in FVC after CS initiation would postpone the moment of reaching a $5 \%$ decline in $\mathrm{FVC} \%$ since diagnosis (while CS does not alter the progressive nature of the disease itself), merely delaying the start of antifibrotic treatment.

As the gains of CS initiation in high BALL fHP patients and/or patients without honeycombing are expected in the early stage of treatment and side-effects become more frequent as treatment duration increases, we believe these data do not support long-term CS treatment. However, multicentre randomised controlled trials are needed to assess this question.

In conclusion, both low BALL and honeycombing presence predict poor outcome and absence of CS treatment effect. In fHP patients with high BALL or honeycombing absence, a marginal increase in FVC was seen, although FVC decline nor $D_{\mathrm{LCO}}$ decline changed after CS treatment initiation.

Laurens J. De Sadeleer $\oplus^{1,2}$, Frederik Hermans ${ }^{1}$, Els De Dycker ${ }^{1}$, Jonas Yserbyt $^{1}$, Johny A. Verschakelen ${ }^{3}$, Eric K. Verbeken $^{4}$, Geert M. Verleden ${ }^{1,2}$, Stijn E. Verleden ${ }^{2}$ and Wim A. Wuyts $\circledast^{1,2}$

${ }^{1}$ Dept of Respiratory Diseases, University Hospitals Leuven, Leuven, Belgium. ${ }^{2}$ Laboratory of Respiratory Diseases, KU Leuven, Leuven, Belgium. ${ }^{3}$ Dept of Radiology, University Hospitals Leuven, Leuven, Belgium. ${ }^{4}$ Dept of Pathology, University Hospitals Leuven, Leuven, Belgium.

Correspondence: Laurens J. De Sadeleer, Katholieke Universiteit Leuven, Laboratory of Respiratory Diseases, Dept of Chronic Diseases, Metabolism and Ageing (CHROMETA), Herestraat 49, box 706, Leuven 3000, Belgium. E-mail: laurens.desadeleer@kuleuven.be

Received: 11 Oct 2019 | Accepted after revision: 26 Dec 2019

Acknowledgements: We thank the patients who participated in this study.

Author contributions: Study design: L.J. De Sadeleer and W.A. Wuyts; data retrieval: L.J. De Sadeleer, E. De Dycker and F. Hermans; data analysis: L.J. De Sadeleer; manuscript writing: L.J. De Sadeleer, S.E. Verleden and W.A. Wuyts; reviewing and editing: all authors.

Conflict of interest: L.J. De Sadeleer reports non-financial support for meeting attendance from Roche and Boehringer Ingelheim, outside the submitted work. F. Hermans has nothing to disclose. E. De Dycker has nothing to disclose. J. Yserbyt has nothing to disclose. J.A. Verschakelen has nothing to disclose. E.K. Verbeken has nothing to disclose. G.M. Verleden has nothing to disclose. S.E. Verleden has nothing to disclose. W.A. Wuyts reports grants from Roche and Boehringer Ingelheim, outside the submitted work.

Support statement: Research reported in this publication was supported by the Research Foundation - Flanders and the University Hospitals Leuven $(1.8 .325 .12 \mathrm{~N})$. The funding sources were not involved in study design, data collection, data analysis, data interpretation, manuscript writing or the decision to submit the article for publication. Funding information for this article has been deposited with the Crossref Funder Registry.

\section{References}

1 Wijsenbeek M, Kreuter M, Olson A, et al. Progressive fibrosing interstitial lung diseases: current practice in diagnosis and management. Curr Med Res Opin 2019; 35: 2015-2024.

2 Kokkarinen JI, Tukiainen HO, Terho EO. Effect of corticosteroid treatment on the recovery of pulmonary function in farmer's lung. Am Rev Respir Dis 1992; 145: 3-5.

3 Vasakova M, Morell F, Walsh S, et al. Hypersensitivity pneumonitis: perspectives in diagnosis and management. Am J Respir Crit Care Med 2017; 196: 680-689.

4 Salisbury ML, Gu T, Murray S, et al. Hypersensitivity pneumonitis: radiologic phenotypes are associated with distinct survival time and pulmonary function trajectory. Chest 2019; 155: 699-711. 
5 De Sadeleer LJ, Hermans F, De Dycker E, et al. Effects of corticosteroid treatment and antigen avoidance in a large hypersensitivity pneumonitis cohort: a single-centre cohort study. J Clin Med 2019; 8: 1-11.

6 Hansell DM, Bankier AA, MacMahon H, et al. Fleischner Society: glossary of terms for thoracic imaging. Radiology 2008; 246: 697-722.

7 Flaherty KR, Wells AU, Cottin V, et al. Nintedanib in progressive fibrosing interstitial lung diseases. $N$ Engl J Med 2019; 381: 1718-1727.

Copyright $@$ ERS 2020 